\title{
The Overall Survival of Patients with Hepatocellular Carcinoma Correlates with the Newly Defined Time to Progression after Transarterial Chemoembolization
}

\author{
Tadaaki Arizumi Kazuomi Ueshima Mina Iwanishi Tomohiro Minami \\ Hirokazu Chishina Masashi Kono Masahiro Takita Norihisa Yada \\ Satoru Hagiwara Yasunori Minami Hiroshi Ida Yoriaki Komeda \\ Mamoru Takenaka Toshiharu Sakurai Tomohiro Watanabe \\ Naoshi Nishida Masatoshi Kudo \\ Department of Gastroenterology and Hepatology, Kindai University Faculty of Medicine, \\ Osaka-Sayama, Japan
}

\section{Keywords}

Hepatocellular carcinoma $\cdot$ Kinki criteria $\cdot$ Overall survival $\cdot$ Time to progression after transarterial chemoembolization · Transarterial chemoembolization

\begin{abstract}
Aim/Background: The ultimate aim of any treatment for hepatocellular carcinoma (HCC) is to improve overall survival (OS); however, the clinical significance of time to progression (TTP) after transarterial chemoembolization (TACE) is unclear. This retrospective study examined the association between OS and the newly defined time to TACE progression (TTTP) to assess whether TTTP can be an alternative to OS in HCC patients with Barcelona Clinic Liver Cancer (BCLC) stage B. Methods: Between January 2006 and December 2013, 592 patients with HCC ( $\mathrm{BCLC} B 1, n=118 ; \mathrm{BCLC} B 2, n=170$ ) underwent TACE. TTTP was then redefined as time to progression from the first image taken after TACE. The relationship between TTTP and OS was then examined based on survival time. Results: Survival analysis revealed significant differences in the OS of patients with BCLC B1 and those with BCLC B2 (median OS: 42.3 months, 95\% confidence interval [CI] 34.4-50.7; and 29.3 months, 95\% CI 26.1-37.6, respectively, $p=$ 0.0348). The median TTTP values were 9.5 months (95\% CI 7.0-10.9) and 5.3 months (95\% CI 4.6-6.7), respectively ( $p=0.0078)$. There was a moderate positive correlation between OS and TTTP for both $\mathrm{B} 1\left(R^{2}=0.6563, p=0.0045\right)$ and $\mathrm{B} 2\left(R^{2}=0.6433, p=0.0052\right)$ substages. There was also a positive correlation between OS and TTTP for the combined B1 and B2 substages
\end{abstract}

Masatoshi Kudo, MD, PhD

Department of Gastroenterology and Hepatology

Kindai University Faculty of Medicine

377-2 Ohno-Higashi, Osaka-Sayama 589-8511 (Japan)

E-Mailm-kudo@med.kindai.ac.jp 
$\left(R^{2}=0.6590, p=0.0024\right)$. Conclusions: There was a moderate correlation between the TTTP and OS of patients with HCC after TACE therapy, where the patients with short TTTP represented short OS, indicating that TTTP is an alternative parameter for survival analysis of HCC patients with BCLC stage $B$ tumors who undergo TACE.

(c) 2017 S. Karger AG, Basel

\section{Introduction}

Despite recent diagnostic and therapeutic advances, hepatocellular carcinoma (HCC) is still the third-most lethal malignancy worldwide and is associated with increasing mortality [1]. Nationwide follow-up surveys conducted by the Liver Cancer Study Group of Japan show that the total number of liver cancer deaths began to increase sharply in 1975, peaked in 2004, and declined again thereafter. It is noteworthy that the establishment of a nationwide HCC surveillance program contributed to improved overall survival (OS) rates [2]. With respect to treatment, sorafenib is effective for cases of advanced HCC [3, 4], and various embolic materials and catheters have been developed for transarterial chemoembolization (TACE) [5]. These advances have contributed to improved OS rates in the past decade. In addition to OS, studies examining the efficacy of anticancer agents and other interventions have examined time to progression (TTP), which is generally defined as the time from initiation of treatment intervention to tumor progression per Response Evaluation Criteria in Cancer of the Liver (RECIST), and is evaluated by comparing pretreatment images with follow-up images.

However, insufficient deposition of lipiodol, which is indicative of TACE failure, may mean that patients undergo further rounds of TACE, irrespective of whether tumor progression per RECIST is detected. In other words, whether or not progression is confirmed, TACE may be repeated in patients that show a progressive disease. Because this means that multiple TACE procedures could be performed even after tumor progression is confirmed, TTP (as a surrogate measure of evaluating survival) is not suitable for patients treated with TACE.

The effects of TACE can also be masked by the emergence of a new lesion, which is considered progressive disease according to the modified RECIST [6] and the Response Evaluation Criteria in Cancer of the Liver (RECICL) criteria [7]. Although TACE is recommended for patients with Barcelona Clinic Liver Cancer (BCLC) stage B HCC [8], a majority of patients undergoing TACE actually harbor multiple intrahepatic tumors or a single large hepatic tumor. Under these conditions, it is easily assumed that these HCCs will have a high frequency of intrahepatic metastasis; also, because of the biological characteristics of HCC, new lesions are likely to appear.

The appearance of new lesions immediately after TACE is indicative of "progression" and so the treatment response will be classified as progressive disease according to the RECIST and mRECIST criteria, despite any favorable response noted in the initially treated tumor. Currently, sorafenib is the only treatment strategy available after failure of TACE $[9,10]$. However, in clinical practice, "progression" is not determined by development of a new small lesion, and switching from TACE to sorafenib or other therapies does not necessarily happen at this point. In such cases, it is conceivable that a favorable response by the treated tumor would improve survival, irrespective of the development of new lesions.

Although many clinical trials of TACE alone or targeted agents in combination with TACE use OS as a primary endpoint, it takes a long time before its effects are proved [11-13]. Furthermore, the OS benefit is diluted by prolonged post-progression survival [14], because post-trial treatments such as intra-arterial infusion chemotherapy, sorafenib, or other trial agents (including regorafenib, lenvatinib, or immunotherapy) can have a chance of marked antitumor effect, resulting in longer post-progression survival irrespective both in the testing 
and control arms. However, TTP can be evaluated over a shorter period of time than OS; therefore, it would be a more desirable endpoint if it actually correlated with OS. To date, no trial except one [15] has used TTP as a primary endpoint because, as mentioned above, it is unsuitable for assessing the effects of TACE therapy [16]. So far, it is generally well known that TTP based on RECIST does not correlate with OS in targeted agent trials probably because treatment in both testing and control arms is often followed by other post-treatment anticancer agents or procedures [17-19]. In case of a TACE combination trial, the dilution effect on OS by post-trial anticancer treatment would be much larger since the OS of BCLC stage B HCC patients is longer than the OS of BCLC stage C HCC patients.

The aim of this study was to ascertain whether the newly defined time to TACE progression (TTTP) is a useful parameter for assessing the effects of TACE on HCC. Thus, we analyzed the correlation between OS and TTTP among HCC patients who underwent conventional TACE (cTACE) therapy.

\section{Materials and Methods}

\section{Patients}

Between January 2006 and August 2013, 592 patients with HCC underwent cTACE as a first-line treatment at Kindai University Hospital. Of these, 288 who had undergone cTACE during their clinical course and met the inclusion criteria were enrolled in this retrospective study. All patients satisfied the diagnostic criteria for HCC set out in the American Association for the Study of the Liver Disease guidelines. Clinicopathological variables, including demographic parameters, tumor staging (based on the number of focal lesions and the maximum diameter of contrast-enhanced [CE] lesions), Child-Pugh score, and BCLC stage, were collected at the time of referral (i.e., prior to treatment). The inclusion criteria were as follows: (1) a diagnosis of HCC based on histological examination, radiologic findings of early enhancement followed by late washout on CE-CT, or dynamic MRI; (2) performance status of 0; and (3) Child-Pugh class A or B preserved liver function. The exclusion criteria were as follows: (1) concomitant antineoplastic treatment and (2) lack of data before and after cTACE due to missing medical records. Patient characteristics are summarized and presented in Table 1. The patient population comprised 213 men and 75 women. Overall, 180 patients (62.5\%) were positive for the antihepatitis C virus antibodies, 27 (9.4\%) were positive for the hepatitis B virus surface antigen, and 81 (28.1\%) were negative for both. 244 patients (84.8\%) were classified as ChildPugh class A. Epirubicin, miriplatin, and cisplatin were used as the TACE chemoagent in 265, 20, and 3 patients, respectively.

\section{Kinki Criteria}

For substaging according to the Kinki criteria, patients were classified according to their Child-Pugh score (5-7 points or 8-9 points) and according to the number and diameter of the tumors using the Milan criteria and the up-to-seven criteria. Substage B1 cases had a Child-Pugh score of 5-7 and met the up-toseven criteria; substage B2 cases had a Child-Pugh score of 5-7 and exceeded the up-to-seven criteria; substage B3 cases had a Child-Pugh score of 8-9 and any tumor status [20-23].

\section{TACE Technique}

The right femoral artery was accessed using an 18-gauge Seldinger needle and a 4-Fr sheath inserted. The celiac artery was catheterized using a 4.2-Fr catheter, through which a 2.2-Fr microcatheter (Shirabe ${ }^{\circledR}$; Piolax, Yokohama, Japan) was advanced coaxially into the common or proper hepatic artery. Rotational angiography was then performed to evaluate the vessels feeding identified tumors. The tip of the catheter was then placed into the segmental and subsegmental feeding arteries with assistance from selective hepatic angiography and/ or tracking navigation imaging. Chemoembolization was then performed using 60-120 mg of miriplatin (Miripla ${ }^{\circledR}$; Sumitomo Dainippon Pharma, Osaka, Japan), 20-50 mg of epirubicin (Epirubicin ${ }^{\circledR}$; Nippon Kayaku, Tokyo, Japan), or 50-100 mg of cisplatin (IA-call ${ }^{\circledR}$; Nippon Kayaku) emulsified in iodized oil (Lipiodol ${ }^{\circledR}$ Ultra- $^{-}$ Fluid; Guerbet, Paris, France) containing gelatin sponge particles (Gelpart ${ }^{\circledR}$; Nippon Kayaku, or Gelfoam ${ }^{\circledR}$; Upjohn, Kalamazoo, MI, USA). The injection volume of the emulsion $(<8 \mathrm{~mL})$ was based on the tumor volume. Neither drug-eluting-bead TACE (DEB-TACE) nor balloon-occluded TACE (B-TACE) were utilized in this study. 
Arizumi et al.: OS of Patients with HCC Correlates with the Newly Defined Time to Progression after Transarterial Chemoembolization

Table 1. Characteristics of hepatocellular carcinoma patients treated with conventional transarterial chemoembolization therapy

\begin{tabular}{lccr}
\hline Characteristic & All patients $(N=288)$ & B1 substage $(n=118)$ & B2 substage $(n=170)$ \\
\hline Age, years & $73(67-77)^{\mathrm{a}}$ & $74(69-77.75)^{\mathrm{a}}$ & $72.5(66.25-77)^{\mathrm{a}}$ \\
Sex & & & \\
$\quad$ Male & $213(74.0)$ & $86(72.9)$ & $127(74.7)$ \\
$\quad$ Female & $75(26.0)$ & $32(27.1)$ & $43(25.3)$ \\
Child-Pugh score & & & \\
$\quad 5$ & $160(55.6)$ & $71(60.2)$ & $89(52.4)$ \\
$\quad 6$ & $84(29.2)$ & $36(30.5)$ & $48(28.2)$ \\
$\quad 7$ & $11(9.3)$ & $33(19.4)$ \\
Virus status ${ }^{\mathrm{b}}$ & $24(15.3)$ & $12(10.2)$ & $15(8.8)$ \\
$\quad$ HBV & $27(9.4)$ & $77(65.3)$ & $52(30.6)$ \\
$\quad$ HCV & $180(62.5)$ & $29(24.6)$ & $37(26.25-54.75)^{\mathrm{a}}$ \\
$\quad$ Virus negative & $31(28.1)$ & $38(22.25-61)^{\mathrm{a}}$ & $348(269-462.5)^{\mathrm{a}}$ \\
Serum ALT, IU/L & $37(25.75-57.25)^{\mathrm{a}}$ & $320.5(242-448.25)^{\mathrm{a}}$ & $13.35(9.8-18.25)^{\mathrm{a}}$ \\
Serum ALP, IU/mL & $334.5(253-454.75)^{\mathrm{a}}$ & $11.7(8.7-15.5)^{\mathrm{a}}$ & $32(8-360)^{\mathrm{a}}$ \\
Serum platelets, $10^{4} / \mu \mathrm{L}$ & $12.7(9.1-16.925)^{\mathrm{a}}$ & $20(6.25-84.75)^{\mathrm{a}}$ & $154(90.6)$ \\
Serum AFP, ng/mL & $25(7-208)$ & $111(94.1)$ & $13(7.6)$ \\
Chemoagents & $265(92.0)$ & $7(5.9)$ & $3(1.8)$ \\
$\quad$ Epirubicin & $20(6.9)$ & $0(0)$ & \\
$\quad$ Miriplatin & $3(1.0)$ & & \\
Cisplatin & & &
\end{tabular}

Values are shown as $n(\%)$, unless otherwise indicated. ALT, alanine aminotransferase, ALP, alkaline phosphatase, AFP, alpha-fetoprotein, HBV, hepatitis B virus, $\mathrm{HCV}$, hepatitis $\mathrm{C}$ virus.

${ }^{a}$ Dispersion variables are shown as median values $(25-75 \%)$. ${ }^{\mathrm{b}}$ Cases testing positive for HBV surface antigen were considered cases of HBV-related hepatocellular carcinoma (HCC); those testing positive for HCV antibody were considered cases of HCV-related HCC.

\section{Time to TACE Progression}

CE-CT or CE-MRI was initially performed at 1 month after TACE therapy to obtain baseline images. These were then compared with images taken every 1-3 months after therapy. To assess the target lesions, the 5 largest tumors were selected from the intrahepatic lesions for the total of maximum diameter of each viable lesion in one dimension measurement. Progression was defined as a $\geq 20 \%$ increase relative to the post-treatment images (baseline images) in the summed diameter of the 5 largest tumors on post-treatment images. The appearance of vascular invasion or extrahepatic spread was also regarded as progression. TTTP was defined as the interval from the day of baseline images after treatment to the time of progression. For example, if a $\geq 20 \%$ increase in the summed diameter was observed on images obtained 1 month after baseline, progression was deemed to have occurred at 1 month after treatment: the TTTP would therefore be 1 month.

\section{Statistical Analysis}

The outcome of interest, OS, was calculated from the date of the first cTACE to the date of death or last clinic follow-up. Patients were followed up until October 2016. OS was calculated using 2 methods. First, institutional electronic medical records were checked to confirm each patient's vital status or date of death, if available. Second, all patients who remained alive and patients of unknown vital status were censored at the last known clinical follow-up date. Kaplan-Meier survival curves were plotted, and the significance of differences was assessed using the log-rank test. Categorical variables were compared using the Bonferronicorrected Mann-Whitney U test. Differences with a two-tailed $p$ value of $<0.05$ were considered statistically significant. To examine relationships between TTTP and OS of the patients, the Pearson correlation test was applied. Any discrepancies between OS and TTTP were identified from correlation plots. All analyses were performed using the SPSS Medical Pack for Windows, version 10.0 (SPSS, Inc., Chicago, IL, USA). 


\section{Liver Cancer}

\begin{tabular}{l|l}
\hline Liver Cancer 2017;6:227-235 & \multicolumn{2}{l}{} \\
\hline DOI: 10.1159/000475777 & $\begin{array}{l}\text { ( 2017 S. Karger AG, Basel } \\
\text { www.karger.com/lic }\end{array}$ \\
\hline
\end{tabular}

Arizumi et al:: OS of Patients with HCC Correlates with the Newly Defined Time to Progression after Transarterial Chemoembolization

Table 2. Relationship between TTTP and OS in patents with B1 substage hepatocellular carcinoma

\begin{tabular}{cllc}
\hline TTTP range & $\begin{array}{l}\text { Patients } \\
(N=118)\end{array}$ & $\begin{array}{l}\text { Median OS }(95 \% \mathrm{CI}), \\
\text { months }\end{array}$ & $\begin{array}{l}\text { 25th-75th percentile } \\
\text { of OS }\end{array}$ \\
\hline 0-5 months & 34 & $27.0(2.2-84.5)$ & $8.5-40.4$ \\
$5-10$ months & 32 & $24.7(5.6-100.4)$ & $13.9-39.4$ \\
$10-15$ months & 21 & $30.2(8.0-108.7)$ & $15.7-50.3$ \\
$15-20$ months & 13 & $47.0(16.0-113.4)$ & $31.4-55.0$ \\
$20-25$ months & 6 & $46.4(23.5-71.1)$ & $39.2-55.3$ \\
$25-30$ months & 3 & $54.5(46.7-88.4)$ & $30.6-71.5$ \\
$30-35$ months & 2 & $34.4(33.4-35.3)$ & $37.4-34.8$ \\
$35-40$ months & 1 & $37.4(37.4-37.4)$ & $44.0-83.1$ \\
$40-45$ months & 4 & $62.7(44.0-88.2)$ & $76.2-102.1$ \\
$45-50$ months & 0 & $89.2(63.2-115.2)$ & \\
$>50$ months & 2 & &
\end{tabular}

TTTP, time to transarterial chemoembolization progression; OS, overall survival; CI, confidence interval.

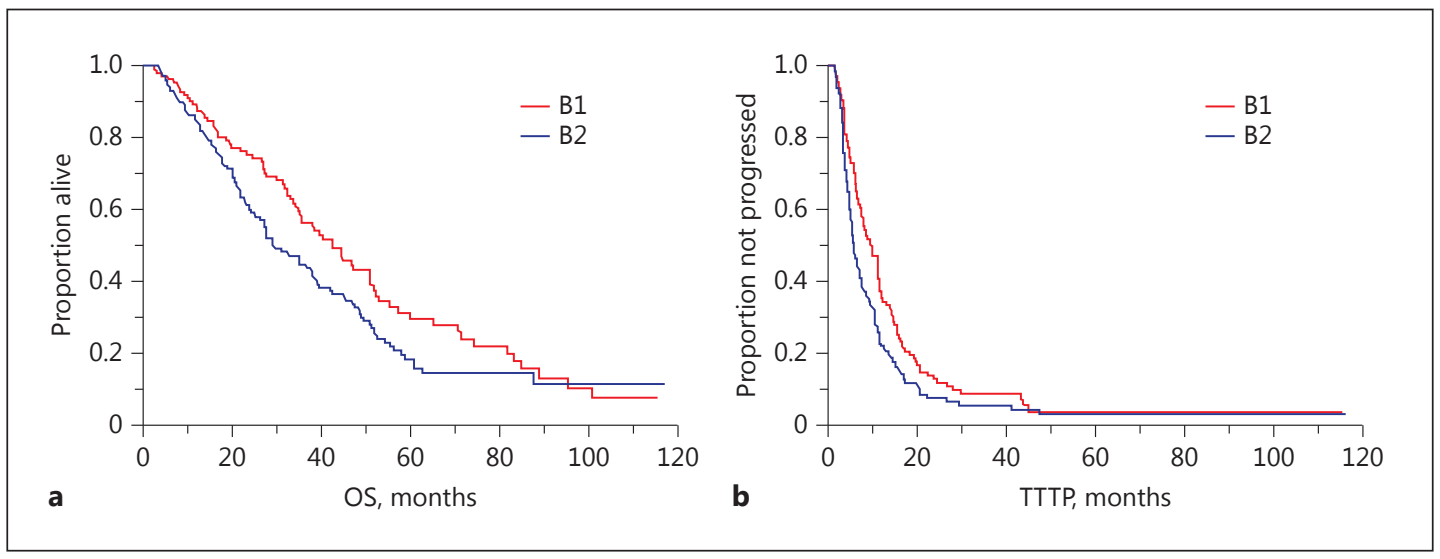

Fig. 1. Comparison of overall survival (OS) (a) and the newly defined time to transarterial chemoembolization progression (TTTP) (b) in patients with B1 and B2 substage hepatocellular carcinoma. a Comparison of survival curves reveals median OS values of 42.3 months (95\% confidence interval [CI] 34.4-50.7) and 29.3 months (95\% CI 26.1-37.6) for the B1 and B2 substage groups, respectively ( $p=0.0348)$. b Comparison of survival curves reveals median TTTP values of 9.5 months (95\% CI 7.0-10.9) and 5.3 months (95\% CI 4.66.7) for the B1 and B2 substage groups, respectively ( $p=0.0078)$.

\section{Results}

\section{OS and TTTP}

The median OS value for BCLC substage B1 and B2 patients combined was 35.3 months (95\% confidence interval [CI] 31.4-41.7). When we compared OS and TTTP among the B1 and B2 substage groups using Kaplan-Meier estimates, we found a significant intergroup difference in the median OS values: 42.3 months (95\% CI 34.4-50.7) for group B1 and 29.3 months (95\% CI 26.1-37.6) for group B2 ( $p=0.0348$ ) (Fig. 1a). Further comparison revealed a significant difference between the groups in terms of the median TTTP, with values of 9.5 months (95\% CI 7.0-10.9) for group B1 and 5.3 months (95\% CI 4.6-6.7) for group B2 ( $p=$ 0.0078) (Fig. 1b). 


\section{Liver Cancer}

Fig. 2. Correlation plots of overall survival (OS) and time to transarterial chemoembolization progression (TTTP). The correlation between OS ( $y$ axis) and TTTP ( $x$ axis) for the B1 (a) and B2 (b) substage groups was assessed separately. A combined analysis was also conducted (c). Each $p$ value shown in the panels was calculated by Bonferroni-corrected Mann-Whitney U test. Both substages demonstrated a positive moderate correlation, with $R^{2}$ values of 0.6563 ( $p=0.0045$ by Pearson correlation test) (a) and 0.6433 ( $p=0.0052$ ) (b) for the B1 and B2 substages, respectively. The combined 2 substages (c) also exhibited a positive correlation with each other $\left(R^{2}=0.6590\right.$, $p=0.0024$ ).

\begin{tabular}{l|l}
\hline \multicolumn{2}{l|}{ Liver Cancer 2017;6:227-235 } \\
\hline DOI: 10.1159/000475777 & $\begin{array}{l}\text { ○ 2017 S. Karger AG, Basel } \\
\text { www.karger.com/lic }\end{array}$ \\
\hline
\end{tabular}

Arizumi et al.: OS of Patients with HCC Correlates with the Newly Defined Time to Progression after Transarterial Chemoembolization
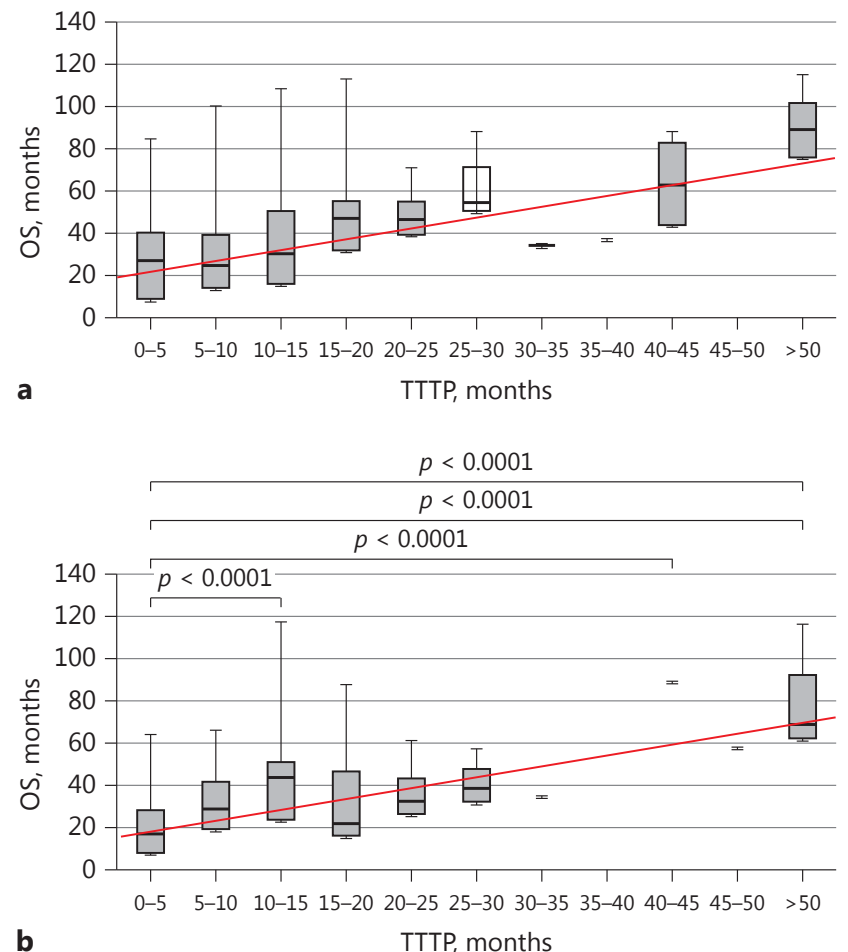

b

TTTP, months

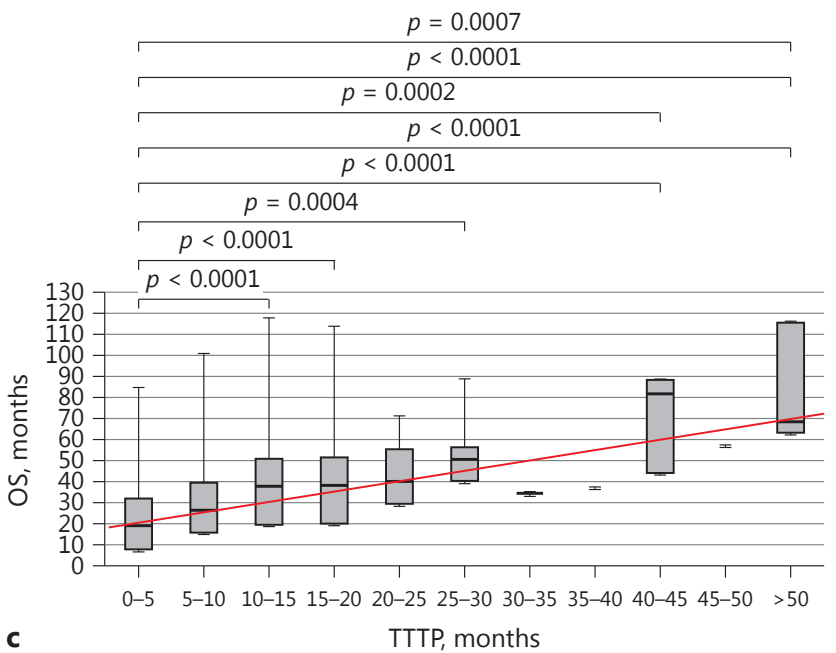

c 
Arizumi et al:: OS of Patients with HCC Correlates with the Newly Defined Time to Progression after Transarterial Chemoembolization

Table 3. Relationship between TTTP and OS in patents with B2 substage hepatocellular carcinoma

\begin{tabular}{clll}
\hline TTTP range & $\begin{array}{l}\text { Patients } \\
(N=170)\end{array}$ & $\begin{array}{l}\text { Median OS }(95 \% \mathrm{CI}), \\
\text { months }\end{array}$ & $\begin{array}{l}\text { 25th-75th percentile } \\
\text { of OS }\end{array}$ \\
\hline $\begin{array}{c}\text { 0-5 months } \\
\text { 5-10 months }\end{array}$ & 81 & $16.9(0.4-63.9)$ & $7.4-27.9$ \\
$10-15$ months & 37 & $28.8(7.7-65.6)$ & $18.8-41.5$ \\
$15-20$ months & 13 & $43.6(11.5-116.9)$ & $23.4-50.7$ \\
$20-25$ months & 4 & $21.5(15.2-87.3)$ & $16.0-46.6$ \\
$25-30$ months & 3 & $32.2(22.4-60.5)$ & $26.0-43$ \\
$30-35$ months & 1 & $38.3(25.3-56.8)$ & $31.8-47.5$ \\
$35-40$ months & 0 & $34.7(34.7-34.7)$ & $88.8-34.7$ \\
$40-45$ months & 1 & $88.8(88.8-88.8)$ & $57.5-57.5$ \\
$45-50$ months & 1 & $57.5(57.5-57.5)$ & $61.9-92.0$ \\
$>50$ months & 3 & $68.2(55.6-115.7)$ & \\
\hline
\end{tabular}

TTTP, time to transarterial chemoembolization progression; OS, overall survival; CI, confidence interval.

Table 4. Relationship between TTTP and OS in patents with B1 and B2 substage hepatocellular carcinoma

\begin{tabular}{cclc}
\hline TTTP range & $\begin{array}{l}\text { Patients } \\
(N=288)\end{array}$ & $\begin{array}{l}\text { Median OS }(95 \% \mathrm{CI}), \\
\text { months }\end{array}$ & $\begin{array}{l}\text { 25th-75th percentile } \\
\text { of OS }\end{array}$ \\
\hline 0-5 months & 115 & $19.4(0.4-84.5)$ & $8.1-32.2$ \\
$5-10$ months & 69 & $26.7(5.6-100.4)$ & $16.3-40.0$ \\
$10-15$ months & 47 & $37.7(8.0-116.9)$ & $19.9-50.5$ \\
$15-20$ months & 26 & $38.4(15.2-113.4)$ & $20.5-51.6$ \\
$20-25$ months & 10 & $40.2(22.4-71.1)$ & $19.6-55.3$ \\
$25-30$ months & 6 & $50.6(25.3-88.4)$ & $34.4-56.2$ \\
$30-35$ months & 3 & $34.7(33.4-35.3)$ & $37.4-37.4$ \\
$35-40$ months & 1 & $37.4(37.4-37.4)$ & $44.1-88.2$ \\
$40-45$ months & 5 & $81.4(44.0-88.8)$ & $57.5-57.5$ \\
$45-50$ months & 1 & $57.5(57.5-57.5)$ & $63.2-115.1$ \\
$>50$ months & 5 & $68.2(55.6-115.7)$ & \\
\hline
\end{tabular}

TTTP, time to transarterial chemoembolization progression; OS, overall survival; CI, confidence interval.

(8.5-40.4), respectively $\left(R^{2}=0.6563, p=0.0045\right)$ (Fig. 2a; Table 2). For the B2 substage group, the corresponding median OS values are presented in Table 3 . A significant difference was observed for the median OS in several comparisons: between the groups with OS of $0-5$ and $10-15$ months ( $p<0.0001), 0-5$ and 40-45 months ( $p<0.0001), 0-5$ and $>50$ months $(p<$ $0.0001)$, and $5-10$ and $>50$ months $(p<0.0001)$ subgroups. In addition, there was a significant positive correlation between OS and TTTP in B2 substages by Pearson correlation analysis $\left(R^{2}=0.6433, p=0.0052\right)$ (Fig. $2 \mathrm{~b}$ ).

The median OS values for each TTTP range when the B1 and B2 substages were combined are presented in Table 4. There was also a significant difference in the median OS between the groups with OS of $0-5$ and $10-15$ months ( $p<0.0001), 0-5$ and 15-20 months $(p<0.0001)$, $0-5$ and $25-30$ months ( $p=0.0004), 0-5$ and $40-45$ months $(p<0.0001), 0-5$ and $>50$ months $(p<0.0001), 5-10$ and $40-45$ months $(p=0.0002), 5-10$ and $>50$ months $(p<0.0001)$, and $10-15$ and $>50$ months ( $p=0.0007)$ subgroups. A significant positive correlation was observed between OS and TTTP for the combined B1 and B2 substages $\left(R^{2}=0.6590 ; p=0.0024\right)$ (Fig. 2c). 


\section{Discussion}

Here, we compared OS and TTTP among patients with substage B1 and B2 HCC. The results according to the Kinki criteria showed that those with substage B1 HCC undergoing cTACE had longer OS and TTTP than those with substage B2 undergoing CTACE. Moreover, OS correlated with TTTP: patients with a longer OS also had a longer TTTP.

Despite administering standard-of-care therapy according to established guidelines, in clinical practice we sometimes encounter patients who present with tumor progression shortly after treatment. These tumors may be resistant to further treatment and the patients often have a poor prognosis.

Early intrahepatic metastasis of HCC may be caused by dissemination of tumor cells via the portal vein [24]. Insufficient deposition of lipiodol may allow TACE-resistant tumors to survive; subsequent dissemination of residual HCC cells via the portal vein leads to early recurrence and a poor prognosis [25]. Here, we assumed that the inefficacy of various posttreatments against the highly malignant tumors meant that patients with a short TTTP also had a short OS.

Currently, many clinical trials of TACE use OS as the primary endpoint because it is not clear whether TTP per RECIST truly reflects OS. Here, we observed median OS values of 42.3 months for the B1 substage group and 29.3 months for the B2 substage group. These median survival times are quite long ( $>2$ years); therefore, any clinical trial that uses OS as an endpoint would require a very long follow-up period. However, the median values for TTTP were 9.5 months for the B1 group and 5.3 months for the B2 group; these times are clearly much shorter and correlate with OS. Thus, the data suggest that TTTP is a suitable endpoint of clinical trials because it requires a shorter follow-up period than OS.

Having said that, a few cTACE-naïve patients showed a discrepancy between OS and TTTP (i.e., some showed a long OS and a short TTTP). One possible reason for this is that a second round of cTACE might have been effective, thereby extending OS. For example, several types of anticancer agents can be used alongside with cTACE. Such second-line chemotherapy agents might have improved the treatment effects. In addition, new TACE procedures such as DEB-TACE (which uses drug-eluting beads) and B-TACE (which uses balloon occlusion techniques) were available for the second round. Another possible reason is that post-cTACE treatment might have been effective, thereby contributing to the long OS observed in some cases with a short TTTP. For example, the use of sorafenib to treat patients with TACErefractory disease and a short TTTP actually prolongs OS $[9,10]$. However, in general we found that OS correlated with TTTP in patients with B1 or B2 substage HCC. Taken together, these results suggest that TTTP can predict OS after TACE. Thus, TTTP is a potential surrogate endpoint for OS in future clinical trials testing the effects of TACE, or new agents combined with TACE, on HCC.

\section{Disclosure Statement}

The authors have no conflicts of interest to declare. 


\section{References}

1 Jemal A, Siegel R, Xu J, Ward E: Cancer statistics, 2010. CA Cancer J Clin 2010;60:277-300.

2 Kudo M: Surveillance, diagnosis, treatment, and outcome of liver cancer in Japan. Liver Cancer 2015;4:39-50.

3 Cheng AL, Kang YK, Chen Z, Tsao CJ, Qin S, Kim JS, Luo R, et al: Efficacy and safety of sorafenib in patients in the Asia-Pacific region with advanced hepatocellular carcinoma: a phase III randomised, double-blind, placebo-controlled trial. Lancet Oncol 2009;10:25-34.

4 Llovet JM, Bruix J: Molecular targeted therapies in hepatocellular carcinoma. Hepatology 2008;48:1312-1327.

5 Minami Y, Minami T, Chishina H, Arizumi T, Takita M, Kitai S, Yada N, et al: Balloon-occluded transcatheter arterial chemoembolization for hepatocellular carcinoma: a single-center experience. Oncology 2015; 89(suppl 2):27-32.

6 Lencioni R, Llovet JM: Modified RECIST (mRECIST) assessment for hepatocellular carcinoma. Semin Liver Dis 2010;30:52-60.

7 Kudo M, Ueshima K, Kubo S, Sakamoto M, Tanaka M, Ikai I, Furuse J, et al: Response Evaluation Criteria in Cancer of the Liver (RECICL) (2015 revised version). Hepatol Res 2016;46:3-9.

8 Bruix J, Reig M, Sherman M: Evidence-based diagnosis, staging, and treatment of patients with hepatocellular carcinoma. Gastroenterology 2016;150:835-853.

9 Ogasawara S, Chiba T, Ooka Y, Kanogawa N, Motoyama T, Suzuki E, Tawada A, et al: Efficacy of sorafenib in intermediate-stage hepatocellular carcinoma patients refractory to transarterial chemoembolization. Oncology 2014;87:330-341.

10 Arizumi T, Ueshima K, Minami T, Kono M, Chishina H, Takita M, Kitai S, et al: Effectiveness of sorafenib in patients with transcatheter arterial chemoembolization (TACE) refractory and intermediate-stage hepatocellular carcinoma. Liver Cancer 2015; 4:253-262.

11 Kudo M, Han G, Finn RS, Poon RT, Blanc JF, Yan L, Yang J, et al: Brivanib as adjuvant therapy to transarterial chemoembolization in patients with hepatocellular carcinoma: a randomized phase III trial. Hepatology 2014; 60:1697-1707.

12 Meyer T, Kirkwood A, Roughton M, Beare S, Tsochatzis E, Yu D, Davies N, et al: A randomised phase II/III trial of 3-weekly cisplatin-based sequential transarterial chemoembolisation vs embolisation alone for hepatocellular carcinoma. Br J Cancer 2013;108:1252-1259.

13 Zhou B, Yan Z, Liu R, Shi P, Qian S, Qu X, Zhu L, et al: Prospective study of transcatheter arterial chemoembolization (TACE) with ginsenoside Rg3 versus TACE alone for the treatment of patients with advanced hepatocellular carcinoma. Radiology 2016;280:630-639.

14 Terashima T, Yamashita T, Takata N, Nakagawa H, Toyama T, Arai K, Kitamura K, et al: Post-progression survival and progression-free survival in patients with advanced hepatocellular carcinoma treated by sorafenib. Hepatol Res 2016;46:650-656.

15 Kudo M, Imanaka K, Chida N, Nakachi K, Tak WY, Takayama T, Yoon JH, et al: Phase III study of sorafenib after transarterial chemoembolisation in Japanese and Korean patients with unresectable hepatocellular carcinoma. Eur J Cancer 2011;47:2117-2127.

16 Llovet JM, Di Bisceglie AM, Bruix J, Kramer BS, Lencioni R, Zhu AX, Sherman M, et al: Design and endpoints of clinical trials in hepatocellular carcinoma. J Natl Cancer Inst 2008;100:698-711.

17 Cainap C, Qin S, Huang WT, Chung IJ, Pan H, Cheng Y, Kudo M, et al: Linifanib versus Sorafenib in patients with advanced hepatocellular carcinoma: results of a randomized phase III trial. J Clin Oncol 2015;33:172-179.

18 Zhu AX, Park JO, Ryoo BY, Yen CJ, Poon R, Pastorelli D, Blanc JF, et al: Ramucirumab versus placebo as secondline treatment in patients with advanced hepatocellular carcinoma following first-line therapy with sorafenib (REACH): a randomised, double-blind, multicentre, phase 3 trial. Lancet Oncol 2015;16:859-870.

19 Llovet JM, Decaens T, Raoul JL, Boucher E, Kudo M, Chang C, Kang YK, et al: Brivanib in patients with advanced hepatocellular carcinoma who were intolerant to sorafenib or for whom sorafenib failed: results from the randomized phase III BRISK-PS study. J Clin Oncol 2013;31:3509-3516.

20 Kudo M: Heterogeneity and subclassification of Barcelona Clinic Liver Cancer Stage B. Liver Cancer 2016;5: 91-96.

21 Arizumi T, Ueshima K, Iwanishi M, Minami T, Chishina H, Kono M, Takita M, et al: Validation of Kinki Criteria, a modified substaging system, in patients with intermediate stage hepatocellular carcinoma. Dig Dis 2016;34: 671-678.

22 Arizumi T, Ueshima K, Iwanishi M, Minami T, Chishina H, Kono M, Takita M, et al: Validation of a modified substaging system (Kinki Criteria) for patients with intermediate-stage hepatocellular carcinoma. Oncology 2015;89(suppl 2):47-52.

23 Kudo M, Arizumi T, Ueshima K, Sakurai T, Kitano M, Nishida N: Subclassification of BCLC B stage hepatocellular carcinoma and treatment strategies: proposal of modified Bolondi's Subclassification (Kinki Criteria). Dig Dis 2015;33:751-758.

24 Sakon M, Umeshita K, Nagano H, Eguchi H, Kishimoto S, Miyamoto A, Ohshima S, et al: Clinical significance of hepatic resection in hepatocellular carcinoma: analysis by disease-free survival curves. Arch Surg 2000;135: 1456-1459.

25 Adachi E, Matsumata T, Nishizaki T, Hashimoto H, Tsuneyoshi M, Sugimachi K: Effects of preoperative transcatheter hepatic arterial chemoembolization for hepatocellular carcinoma. The relationship between postoperative course and tumor necrosis. Cancer 1993;72:3593-3598. 